\title{
Using LiF crystals for high-performance neutron imaging with micron-scale resolution
}

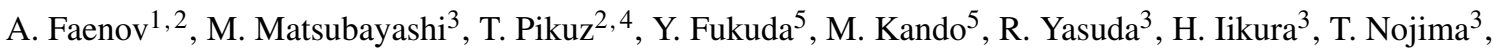 \\ T. Sakai ${ }^{3}$, M. Shiozawa ${ }^{6}$, R. Kodama ${ }^{1,4}$, and Y. Kato ${ }^{7}$ \\ ${ }^{1}$ Institute for Academic Initiatives, Suita, Osaka University, 565-0871, Japan \\ ${ }^{2}$ Joint Institute for High Temperatures, Russian Academy of Sciences, Izhorskaja Street 13/19, Moscow, Russia \\ ${ }^{3}$ Quantum Beam Science Center, Japan Atomic Energy Agency, Tokai, Ibaraki 319-1195, Japan \\ ${ }^{4}$ PPC and Graduate School of Engineering, Suita, Osaka University, 565-0871, Japan \\ ${ }^{5}$ Quantum Beam Science Center, Japan Atomic Energy Agency, Kyoto 619-0215, Japan \\ ${ }^{6}$ Nippon SOKEN, Inc., Iwaya 14, Shimohasumi, Nishio, Aichi 445-0012, Japan \\ ${ }^{7}$ The Graduate School for the Creation of New Photonics Industries, Hamamatsu, Shizuoka 431-1202, Japan \\ (Received 5 March 2015; revised 26 August 2015; accepted 30 September 2015)
}

\begin{abstract}
This paper describes an overview of our recent discovery - clear demonstration that LiF crystals can be efficiently used as a high-performance neutron imaging detector based on optically stimulated luminescence of color centers generated by neutron irradiation. It is shown that the neutron images we have obtained are almost free from granular noise, have a spatial resolution of $\sim 5.4 \mu \mathrm{m}$ and a linear response with a dynamic range of at least $10^{3}$. The high contrast and good sensitivity of LiF crystals allow us to distinguish two holes with less than $2 \%$ transmittance difference. We propose to use such detectors in areas where high spatial resolution with high image gradation resolution is needed, including diagnostics of different plasma sources such as laser and z-pinch produced plasmas.
\end{abstract}

Keywords: color centers; LiF crystals; neutron imaging; neutrons; plasma diagnostics

\section{Introduction}

Typically, neutron radiography ${ }^{[1]}$ with a high spatial resolution tries to solve two types of tasks: (i) to perform selfimaging of large-size (Figure 1a) or tiny (Figure 1b) neutron sources and (ii) to perform imaging of the internal structure of matter by neutron absorption (Figure 1c). Regardless of the scientific or industrial application, there is a requirement to develop neutron detectors with a spatial resolution improvement of up to $5-10 \mu \mathrm{m}$ and with a high sensitivity (few percent) to variation in material thickness and structure. For example, in the development of new electrical devices ${ }^{[2-5]}$ and for neutron source reconstruction from pinhole imaging developed for inertial confined fusion (ICF) experiments using laser irradiation ${ }^{[6-9]}$, a spatial resolution on the scale of a few microns is needed.

Unfortunately, the current best available neutron detectors allow a spatial resolution of only around $15-20 \mu \mathrm{m}$ or worse to be reached. For example, the neutron imaging

Correspondence to: A. Faenov, Institute for Academic Initiatives, Suita, Osaka University, 565-0871, Japan. Email: faenov.anatoly@photon.osakau.ac.jp plate, where the neutron converter $\left(\mathrm{Gd}_{2} \mathrm{O}_{3}\right)$ is mixed with the photo-stimulated luminescence material $\left(\mathrm{BaFBr}_{\mathrm{Eu}}{ }^{2+}\right)$, has high sensitivity ${ }^{[10]}$, with a measured line spread function of $58 \mu \mathrm{m}^{[11]}$. Neutron images have also been recorded with Gd- or B-doped microchannel plates with cross-delay line readout, resulting in an estimated spatial resolution of $17 \mu \mathrm{m}^{[12]}$. Real-time neutron imaging has been demonstrated by projecting the fluorescence from a LiZnS scintillator onto a high-sensitivity CCD camera ${ }^{[13]}$. Recently, dynamic neutron images have been recorded with a neutron color image intensifier, enabling real-time observation of dynamic phenomena with 30 frames/s video pictures, at a thermal neutron flux of $1.5 \times 10^{8} \mathrm{n} /\left(\mathrm{cm}^{2} \mathrm{~s}\right)^{[14]}$. Recently, great progress in the development of large neutron imaging systems for ICF has allowed $15 \mu \mathrm{m}$ spatial resolution to be reached in a wide field of view ${ }^{[8]}$. In such a case neutrons transmitted by the aperture are converted to visible light in a scintillator array that is subsequently recorded by a CCD camera. To achieve a high spatial resolution in such experiments a large magnification is needed because the spatial resolution of the detector is $\sim 650 \mu \mathrm{m}$. 
(a)

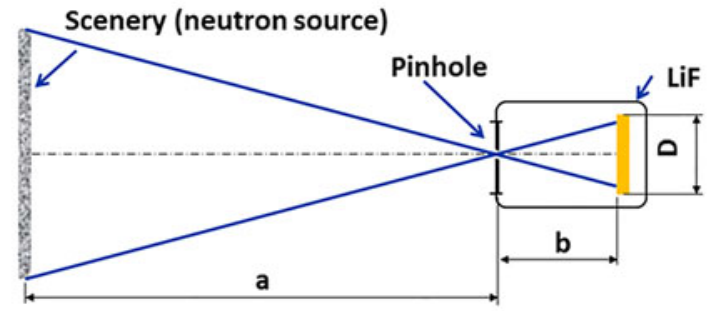

(b)

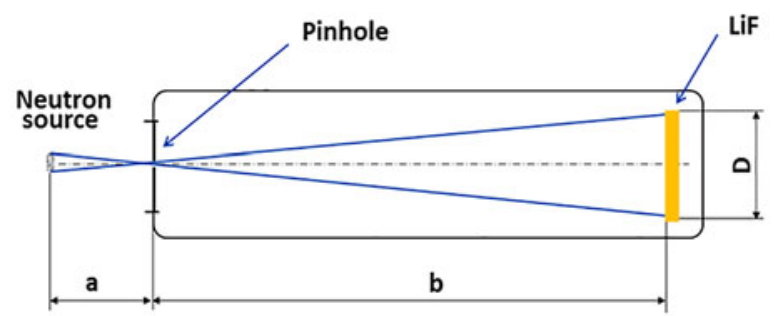

(c)

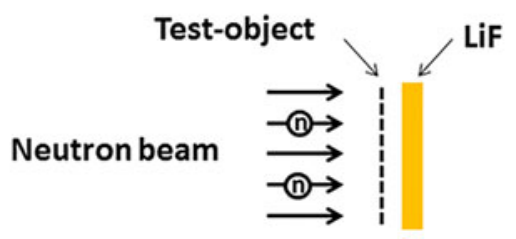

Figure 1. Schematic layouts for neutron radiography by LiF detector. Selfradiography of (a) large-size and (b) tiny neutron sources using a pinhole imaging approach and high-resolution LiF crystal detectors. (c) Neutron radiography of the internal structure of objects. In such a case the object is placed in close contact to the LiF crystal.

At the same time, it is well known that point defects or, as they are also called, color centers (CCs) are produced sufficiently easily under interaction of particles or photons with $\mathrm{LiF}$ crystal ${ }^{[15]}$. Such CCs could be hosted in $\mathrm{LiF}$ at room temperature for a very long time and then under excitation by UV radiation the CCs would emit photoluminescence (PL) in the visible spectral range and allow submicron spatial resolution to be reached for soft x-ray imaging when the penetration depth of the photons is on the scale of tens or hundreds of nanometers. Recently, LiF crystal and film detectors have been successfully used for high-performance soft $\mathrm{x}$-ray conventional and phase-contrast imaging ${ }^{[16-22]}$ of different nanofoils or biological objects. They have also started to be widely used for characterization intensity profiles or focusing properties of $\mathrm{x}$-ray laser or high-order harmonic beams ${ }^{[23-30]}$. Dislocations, point defect clusters and cavities in crystals irradiated by neutrons have been investigated already for many decades ${ }^{[31,32]}$, but only recently we proposed to use $\mathrm{LiF}$ crystals as highperformance neutron imaging detectors ${ }^{[33-36]}$. In this paper we will give an overview of our main results performed for characterization of such a detector and its use as a highspatial-resolution thermal neutron radiography and discuss the applications of such a detector in areas where a high spatial resolution with a high image gradation resolution is needed. We also discuss possible applications of $\mathrm{LiF}$ imaging detectors for diagnostics of different continuous and pulsed high-intensity neutron sources, including plasma sources such as laser and z-pinch produced plasma.

\section{Principles of neutron imaging generation in LiF crys- tals and experimental procedure}

In $\mathrm{LiF}$, the $\mathrm{F}^{3+}$ and $\mathrm{F}^{2} \mathrm{CCs}$ are formed by aggregation of the $\mathrm{F}$ centers, which are produced by irradiation of ionizing radiation. In our case of neutron beam irradiation (see Figures 2 and 3a) the following reaction takes place with a cross-section of 940 barns for thermal neutrons: ${ }^{6} \mathrm{Li}+$ $\mathrm{n} \rightarrow \alpha(2.05 \mathrm{MeV})+{ }^{3} \mathrm{H}(2.73 \mathrm{MeV})$, where the energies of the reaction products are shown in parentheses ${ }^{[37]}$. It is necessary to stress that natural lithium is composed of $7.40 \%$ ${ }^{6} \mathrm{Li}$ and $92.60 \%{ }^{7} \mathrm{Li}$, and only ${ }^{6} \mathrm{Li}$ is involved in the processes mentioned above. The ranges of $\alpha$ and ${ }^{3} \mathrm{H}$ in $\mathrm{LiF}$ are estimated to be $\sim 6$ and $\sim 33 \mu \mathrm{m}$, respectively. This means that that the spatial resolution of $\mathrm{LiF}$ for neutron imaging is determined mainly by the range of the particle propagation inside the LiF crystal. Using such propagation ranges it is possible to estimate that the $\alpha$ and ${ }^{3} \mathrm{H}$ particles will deposit energy of approximately 140 and $33 \mathrm{eV}$ in each lattice cell of the $\mathrm{LiF}$ crystal, respectively. In $\mathrm{LiF}$, the $\mathrm{F}$ centers are created by excitation of valence electrons to the conduction band with an excitation energy ${ }^{[15,16]}$ of $14 \mathrm{eV}$. It follows that the energy deposited by the $\alpha$ particles is sufficient to create many $(\sim 10) \mathrm{F}$ centers simultaneously in each lattice, leading to efficient formation of the $\mathrm{F}^{3+}$ and $\mathrm{F}^{2} \mathrm{CCs}$ (Figure 2).

The thermal neutron radiography facility (TNRF-2) at the research reactor JRR-3M (20 MW thermal output) at JAEA was used for micron-scale neutron imaging in our experiments. The beam line for TNRF-2 provides ${ }^{[38]}$ thermal neutrons (peak energy of approximately $30 \mathrm{meV}$ ) with a flux of $1.0 \times 10^{8} \mathrm{n} /\left(\mathrm{cm}^{2} \mathrm{~s}\right)$, and the $L / D$ ratio varies from 100 to 460 , where $L$ is the distance from the reactor to the sample and $D$ is the aperture size at the reactor. In the TNRF-2 station, the dose rate of the gamma rays has been measured to be $2.16 \mathrm{~Sv} / \mathrm{h}$. The image recording and acquisition setup for the neutron imaging experiments using $\mathrm{LiF}$ crystals is shown in Figure 1(c). In image recording, the objects were placed in close contact to the $\mathrm{LiF}$ crystal mounted in an $\mathrm{Al}$ holder and covered with an $\mathrm{Al}$ foil. A LiF crystal of $20 \mathrm{~mm}$ diameter and $3 \mathrm{~mm}$ thickness, polished on both sides, was used in our experiments. The procedures for neutron irradiation of different samples and the readout process were described in detail in Refs. [33-36].

After recording, the images of the neutron beam intensity distribution created inside the $\mathrm{LiF}$ crystal by $\mathrm{F}^{3+}$ and $\mathrm{F}^{2} \mathrm{CCs}$ were read out using a laser confocal fluorescence microscope (Olympus model FV-300), as shown in Figure 3(b). The $\mathrm{LiF}$ crystal under the microscope was illuminated with the $488 \mathrm{~nm}$ line of an argon ion laser and the luminescence 


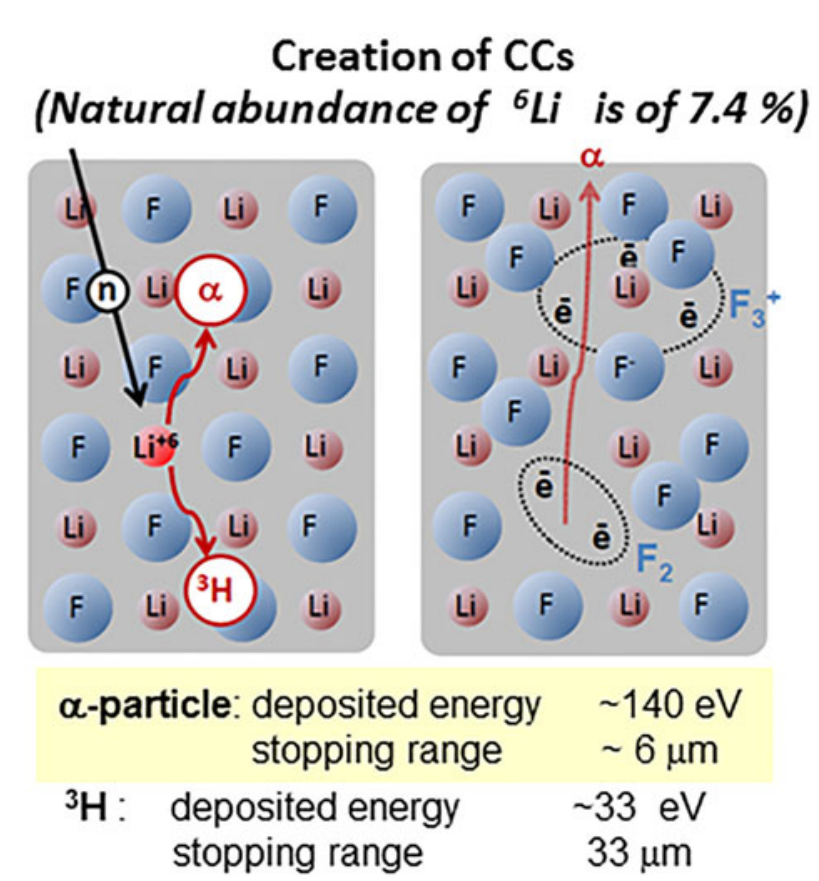

Figure 2. Principles of neutron imaging generation in LiF crystals.

from the CCs at $>510 \mathrm{~nm}$ was observed. Different samples were used for characterization of the LiF crystal as a highperformance neutron imaging detector.

\section{Features of LiF crystal as a neutron imaging detector}

Several important parameters of the $\mathrm{LiF}$ neutron imaging detector were characterized during our experiments.

\subsection{Spatial resolution}

The spatial resolution of the LiF crystal neutron imaging detector was quantitatively evaluated by two different approaches. First of all, we used specially produced masks, which consisted of line pair patterns fabricated on a
$0.005 \mathrm{~mm}$ thick Gd film evaporated on a glass plate ${ }^{[36]}$. The images of the small line pairs on the dark field were observed using a LiF single crystal detector and are presented in Figure 4. From this figure, splits in the small line pairs as little as $10 \mu \mathrm{m}$ wide are clearly seen with good contrast in the images. This result demonstrates that the LiF crystal neutron detector has potential for use in the practical evaluation of the spatial resolution of noble gas imaging having an ultra-high spatial resolution of $\sim 5 \mu \mathrm{m}$.

As a second approach ${ }^{[33]}$, we studied the spatial resolution of the LiF neutron detector by measuring the sharpness of the edges for low and heavy neutron absorbed materials. In Figure 5 the neutron images of the edges of a $100 \mu \mathrm{m}$ thick Cd (low-neutron-absorption material) and a $100 \mu \mathrm{m}$ thick Gd (high-neutron-absorption material) plate are presented. The radiography image of the $100 \mu \mathrm{m}$ thick Cd plate was recorded at only $10 \mathrm{~s}$ neutron exposure. Comparison of this image with a Gaussian error function shows that the best fit to the experiment corresponds to a spatial resolution of $\sim 5.4 \mu \mathrm{m}$, where the spatial resolution is defined as the distance between $10 \%$ and $90 \%$ of the transmittance of the fitted curve. This spatial resolution agrees well with the estimated stopping range of $\alpha$ particles in $\operatorname{LiF}(\sim 6 \mu \mathrm{m})$. The neutron images of the $100 \mu \mathrm{m}$ thick Gd plate (see Figure 5b) match the case when almost complete absorption of the thermal neutrons with a transmittance of only $3.6 \times 10^{-7}$ takes place. The neutron image of the Gd plate shown in Figure 5(b) is compared with the optical microscope image and it is clearly demonstrated that the detailed structures seen in the optical image are well reproduced in the neutron image. We should stress that small structures with a very tiny width are well resolved in the neutron image. Moreover, the trace of the magnified part of the image of a small crack presented in Figure 5(b) gives a value of the spatial resolution of $\sim 5.5 \mu \mathrm{m}$, which is in good consistency with the $\sim 5.4 \mu \mathrm{m}$ spatial resolution estimated in Figure 5(a) for low-neutron-absorption material. (a)

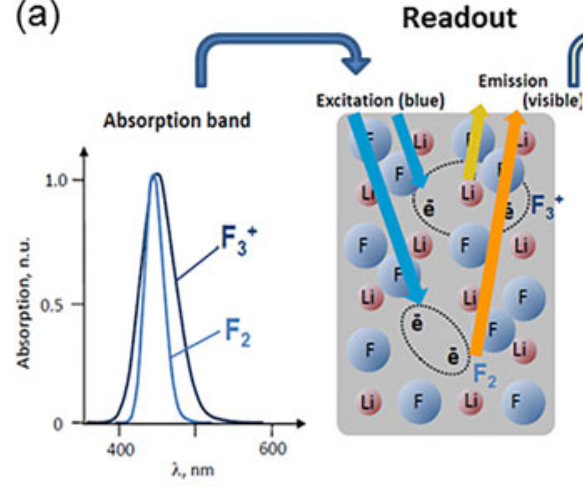

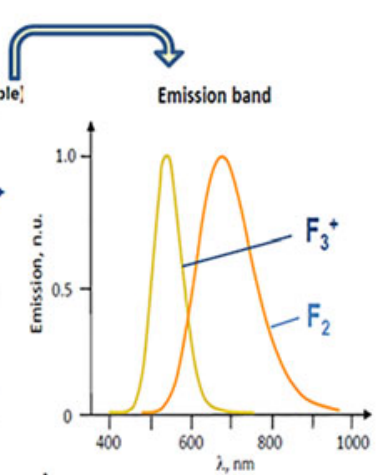

(b)
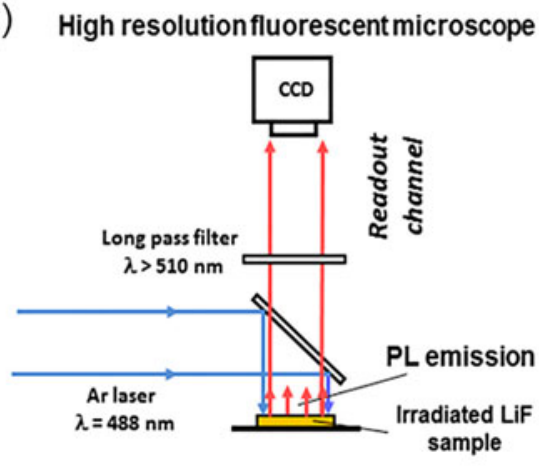

Figure 3. In image readout, luminescence from the LiF crystal was observed with a laser scanning confocal luminescence microscope. The $\lambda=488 \mathrm{~nm}$ line of an argon laser was used for excitation and luminescence from the CCs at $\lambda>510 \mathrm{~nm}^{[33-36]}$. 


\section{(unit: $\mathrm{mm}$ )}

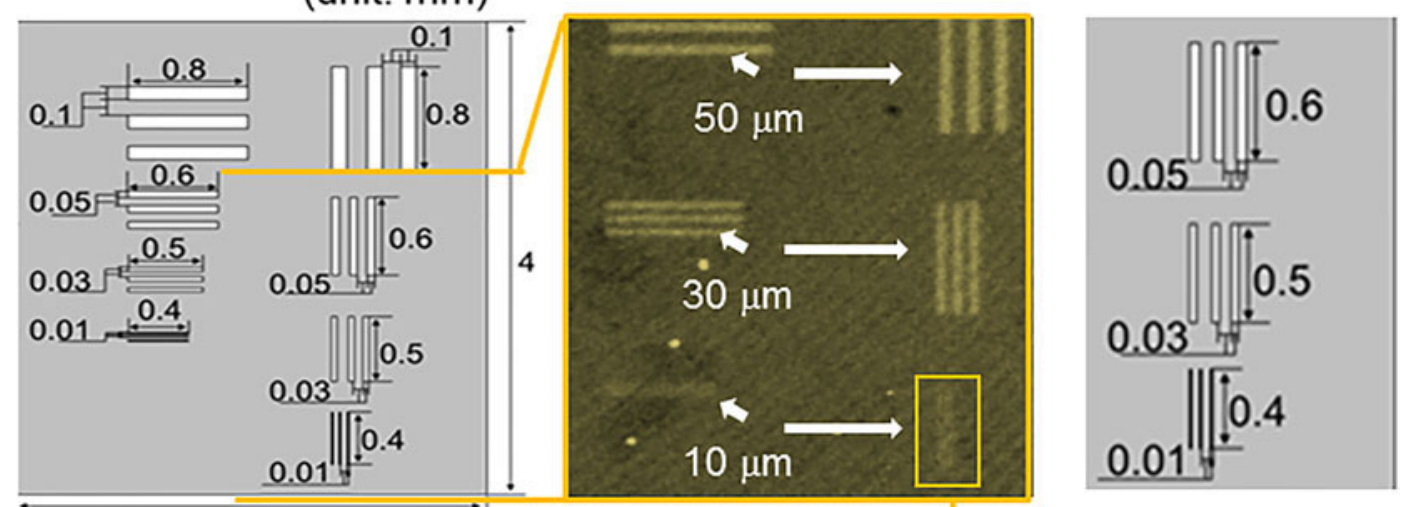

2.5

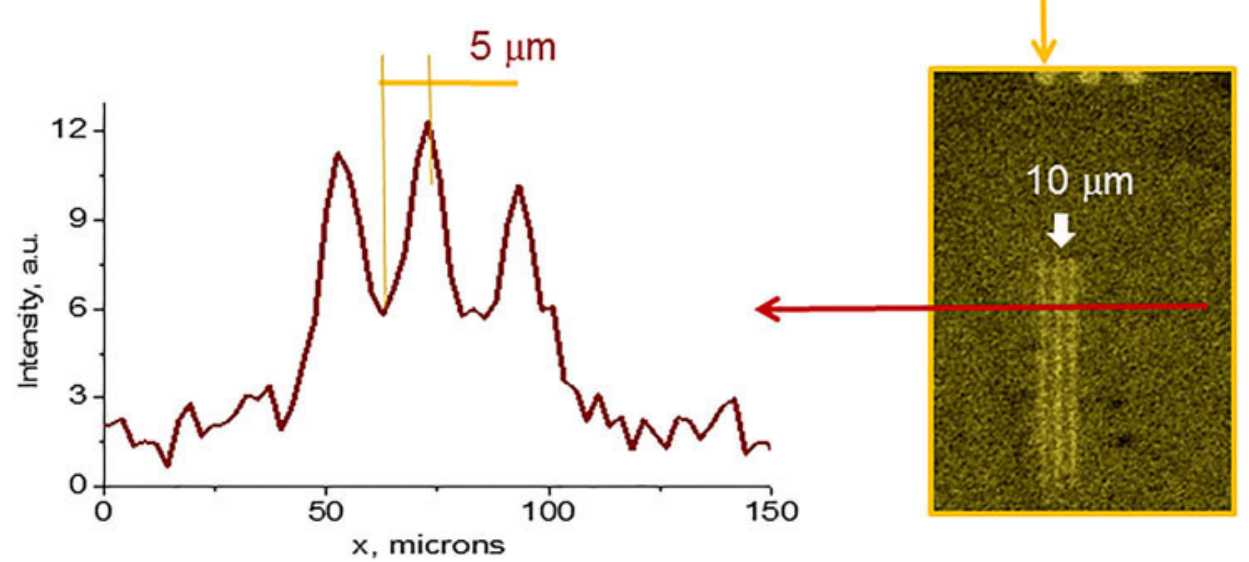

Figure 4. Schematic diagrams and sizes of the line pairs produced on $5 \mu \mathrm{m}$ thickness Gd patterns coated onto the overall surface of a glass substrate and their images obtained by using the LiF crystal neutron imaging detector (top). Line-pair images obtained using the LiF single crystal detector and line profiles of the pairs with widths of $10 \mu \mathrm{m}^{[36]}$ (bottom). The spatial resolution on the scale of $5 \mu \mathrm{m}$ is clearly seen.

\subsection{Sensitivity and linearity of the LiF crystal imaging detector}

Other very important characteristics of any detector are the sensitivity and linearity of the detector response to different fluxes of incoming radiation intensity. We conducted different experiments ${ }^{[33]}$ to measure these important parameters. Figure 6(a) shows the neutron images of Au wires of 42, 95 and $287 \mu \mathrm{m}$ diameter, recorded at 10 and $30 \mathrm{~min}$ exposure, corresponding to neutron fluences of $6 \times 10^{10}$ and $1.8 \times$ $10^{11} \mathrm{n} / \mathrm{cm}^{2}$, respectively. It is clearly seen from Figure $6(\mathrm{a})$ that all of the three wires are resolved, including the smallest wire of $42 \mu \mathrm{m}$ diameter, which has an attenuation of only $2.35 \%$. This demonstrates that LiF has not only high spatial resolution but also high sensitivity, high imaging contrast and, which is very important for neutron imaging detectors, the image quality is free from granular noise that sometimes exists in other neutron imaging detectors. Indeed, for the data presented in Figure 6(a) the signal-to-noise ratio has a very high value of $\sim 50$ for the thinnest $42 \mu \mathrm{m}$ diameter $\mathrm{Au}$ wire. As seen in Figure 6(b), there is a very good agreement between the experimental data and the theoretical curves for the neutron transmittance across the radial direction of the wires in the case of using the theoretical transmittance $\mu=5.66$ (bottom panel). This transmittance corresponds to the energy of the thermal neutrons applied for the irradiation of the Au wires. We would like to stress that a very small change of the neutron transmittance coefficient value, for example to $\mu=6.36$, could be clearly distinguished by comparison with experimental curves (see the upper panel in Figure 6b). This means that if the energy of the thermal neutrons is known with high accuracy the transmittance coefficient value can be measured with high accuracy.

Additional proof of the high spatial resolution, high sensitivity and high contrast of neutron imaging by LiF crystal detectors in the case of imaging of high-neutron-absorption materials is obviously given by Figure 7. In this experiment the gadolinium plate, which was hammered during cutting by scissors, was irradiated by a neutron beam for $30 \mathrm{~min}$. The obtained image shows that changes of thickness of the hammered Gd plate from approximately 0 to $\sim 25 \mu \mathrm{m}$ could be measured with an accuracy of some microns. Additionally, all tiny defects on the surface and edge of the foil (for example, such as the burr in Figure 7) could be evidently distinguished with a resolution of a few microns and measured. 
(a)
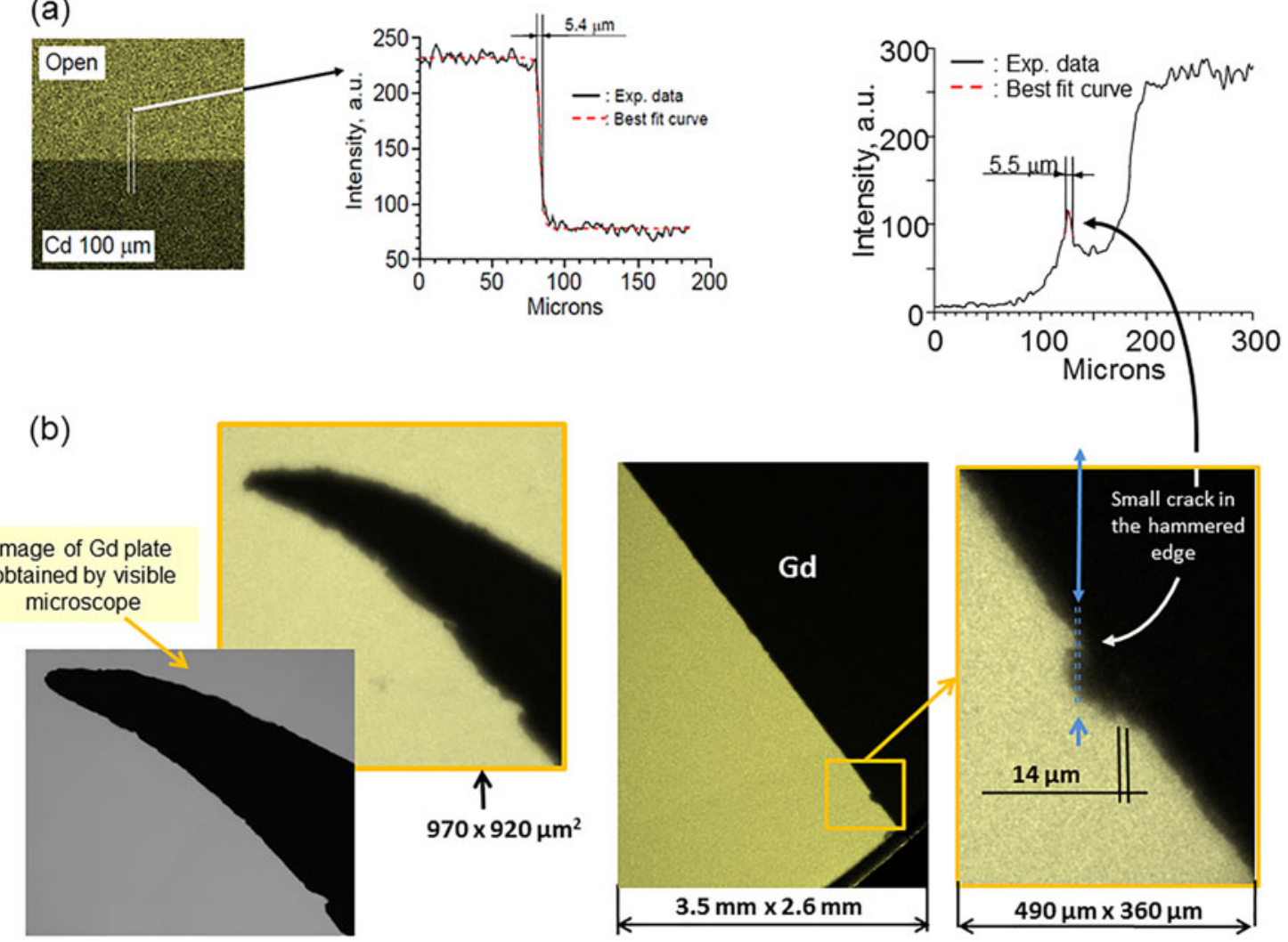

Figure 5. (a) Neutron image of a $100 \mu \mathrm{m}$ thick Cd plate taken with $10 \mathrm{~s}$ exposure time and a trace of the neutron image across the edge, which is compared with a calculation at a spatial resolution of $5.4 \mu \mathrm{m}^{[33]}$. (b) Neutron radiography images of a $100 \mathrm{~mm}$ thick Gd plate of triangular shape ${ }^{[33]}$. Optical microscope and neutron images of the same part near the edge obviously demonstrate a high-resolution quality of the LiF neutron imaging detector comparable with optical microscopy imaging. The magnified image of a small crack in the Gd plate and the line scan of this part, shown by the blue lines, clearly manifest high contrast and spatial resolution of such images. We could see that this line scan has a best fit with a modeled curve with a $5.5 \mu \mathrm{m}$ width (dashed curve).

(a)
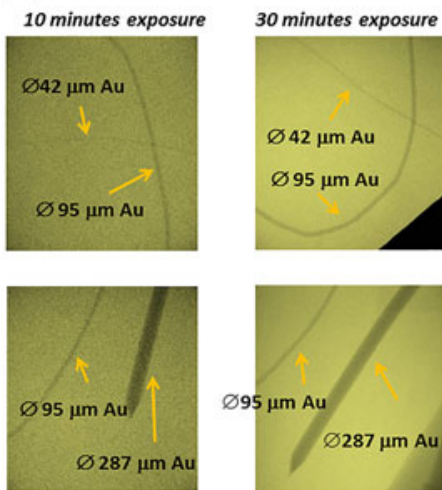

$42 \mu \mathrm{m}$ Gold wire with absorption only about of $2.35 \%$ is clearly imaged!!

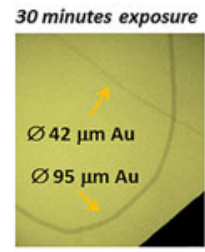

(b) The sensitivity of neutron imaging modeling from attenuation of neutron beam coefficient:

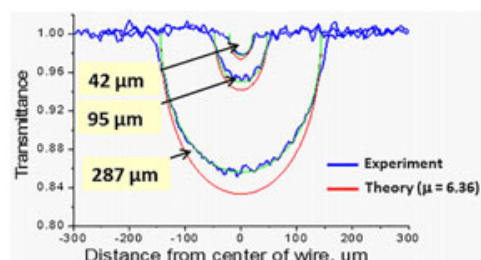

Corrected result:

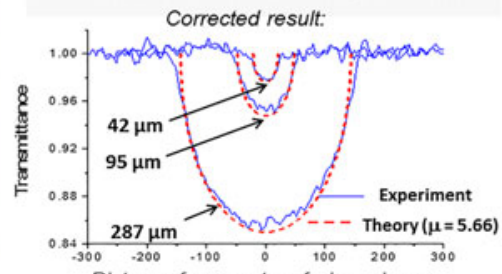

(c)

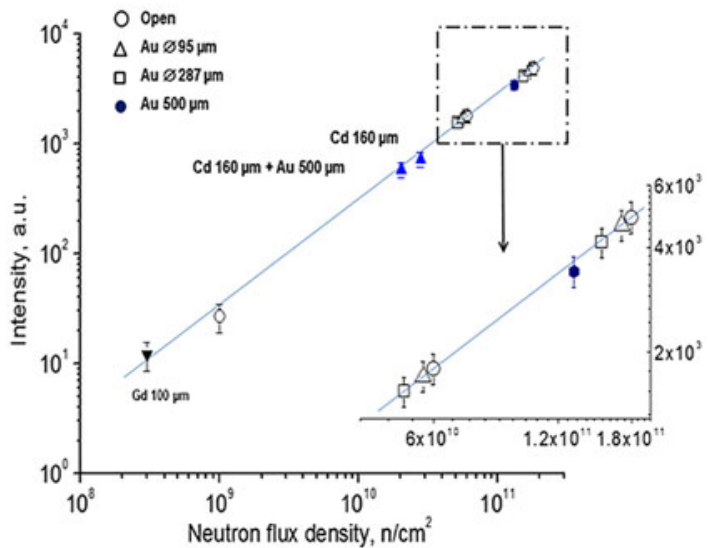

Figure 6. (a) Comparison of the neutron images of the Au wires of 42,95 and $287 \mu \mathrm{m}$ diameter recorded with exposure times of 10 and 30 min ${ }^{[33]}$. (b) Comparison ${ }^{[33]}$ of the traces of the experimental intensity transmittance of neutrons through the Au wires (solid curves) with the theoretical transmittance (dashed curves) for two attenuation beam coefficients. It is clearly seen that the best coincidence between the modeling and the experimental curves is obtained for $\mu=5.66 \mathrm{~cm}^{-1}$ (bottom panel). Changes of $\mu$ of even $\sim 12 \%\left(\mu=6.36 \mathrm{~cm}^{-1}\right)$ show a large disagreement between the theoretical and experimental curves, which testifies to the high quality and sensitivity of the LiF crystal neutron imaging detector. (c) A plot of the luminescent intensity from the CCs in $\mathrm{LiF}$ versus the neutron fluence on $\mathrm{LiF}^{[33]}$. The neutron fluence was varied by the neutron exposure time and the attenuation of the neutron flux by various filters, such as Au wires, Au foils and Cd plates. The straight line is a fit to the data, showing a good linear response of the LiF to the neutron fluence. 


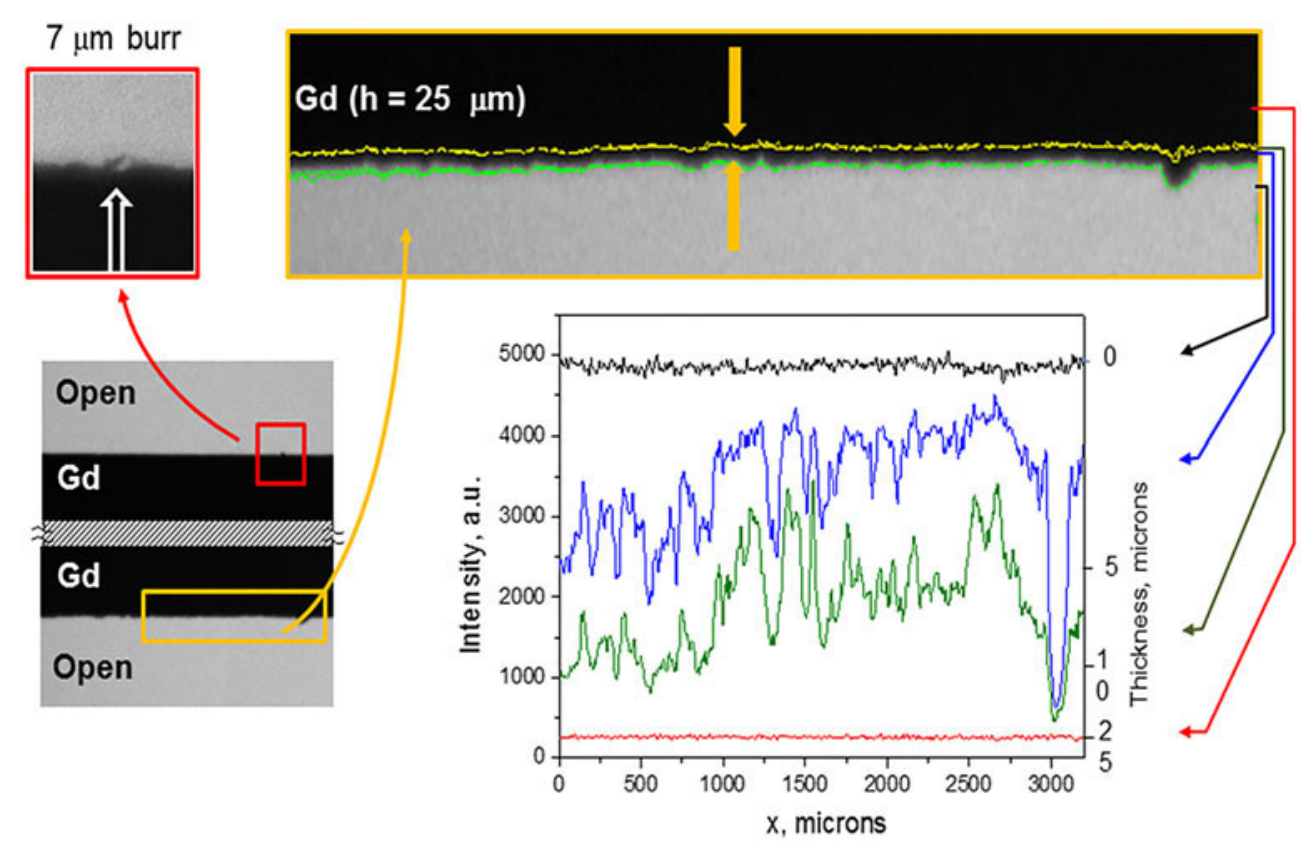

Figure 7. Neutron radiography of a $25 \mu \mathrm{m}$ thick Gd plate. A defect with a size of $\sim 7 \mu \mathrm{m}$ and some micron-scale changes of thickness of the hammered Gd plate edge (due to cutting the Gd plate with scissors) are clearly seen in the magnified images of different parts of the sample ${ }^{[35]}$.

The linearity of the LiF detector was checked (see Figure $6 \mathrm{c}$ ) by comparing the luminescence intensities of the images irradiated with the different neutron fluences which were varied by the exposure time $(30 \mathrm{~min}, 10 \mathrm{~min}$ and $10 \mathrm{~s})$ and the attenuation of neutron intensity by various materials ( $\mathrm{Au}$ wires, $\mathrm{Au}$ foils and $\mathrm{Cd}$ plates). From this figure we can see that the luminescence intensity is highly linear to the neutron fluence over almost three orders of magnitude ${ }^{[33]}$, starting from $3 \times 10^{8}$ to $1.8 \times 10^{11} \mathrm{n} / \mathrm{cm}^{2}$. It is demonstrated that the $\mathrm{LiF}$ crystal imaging detector has a very high dynamic range similar to the dynamic range of LiF crystal for $\mathrm{x}$-ray imaging ${ }^{[16]}$. We should stress that luminescence, produced by neutron irradiation, is observed even for the area covered with highly attenuating filters (see Figure 6c). From the measured noise of the neutron images, the minimum detection level of neutrons with $\mathrm{LiF}$ was estimated to be approximately $2.5 \times 10^{8} \mathrm{n} / \mathrm{cm}^{2}$. This neutron fluence corresponds to the $\sim 2.5 \mathrm{~s}$ exposure time of the JRR$3 \mathrm{M}$ neutron facility used in these experiments ${ }^{[38]}$. Indeed, we could clearly record the neutron images with 10 seconds exposure, as shown in Figure 5(a). It is necessary to mention that the neutron detection efficiency of LiF is partly limited by the absorption of the neutrons in LiF. Actually, since the attenuation coefficient of thermal neutrons in $\mathrm{LiF}$ is estimated to be $5.2 \mathrm{~cm}^{-1}$, only $0.26 \%$ of the neutrons are absorbed in the $\sim 5 \mu \mathrm{m}$ depth of LiF, which corresponds to the focal depth of the $20 \times$ microscope objective. To check this point we read out neutron images not only from the front side but also from the rear side of the $3 \mathrm{~mm} \mathrm{LiF}$ crystal. In this case clear neutron images were recorded from both sides of the crystal. The intensity of the image from the rear of the
LiF crystal was sufficiently good, but the spatial resolution and contrast of this image were worse compared with the image that was read out from the front. This occurred due to the scattering when neutrons pass through the $3 \mathrm{~mm}$ thickness of the LiF crystal.

Additional metrological testing of the LiF crystal neutron imaging sensitivity provided in Ref. [34] by using the standard neutron sensitivity indicator shows that holes with transmittance differences of less than $2 \%$ could be observed. Moreover, all gaps in this indicator with sizes from 22 to $26 \mu \mathrm{m}$ were also clearly observed. All of the above mentioned experimental results show that LiF crystals have excellent characteristics as neutron imaging detectors with high spatial resolution, high dynamic range and good contrast.

\section{Imaging of samples with internal gas or water struc- tures using the LiF neutron imaging detector}

One of the main advantages of thermal neutron imaging in comparison to $x$-ray imaging is the capability of observing materials comprising high- and low- $Z$ elements. In Figure 8 (a) neutron imaging ${ }^{[33]}$ of a ball-point pen in which liquid ink is contained in a thin metal tube is presented. The high contrast and good spatial resolution of the obtained image allow the change of the metal tube diameter from $\sim 370 \mu \mathrm{m}$ diameter at the smallest part of the pen to $\sim 1040 \mu \mathrm{m}$ diameter at the largest to be distinguished. The metallic parts such as the ink tube and the roller ball at the tip are obviously distinguished too. Furthermore, 
(a)

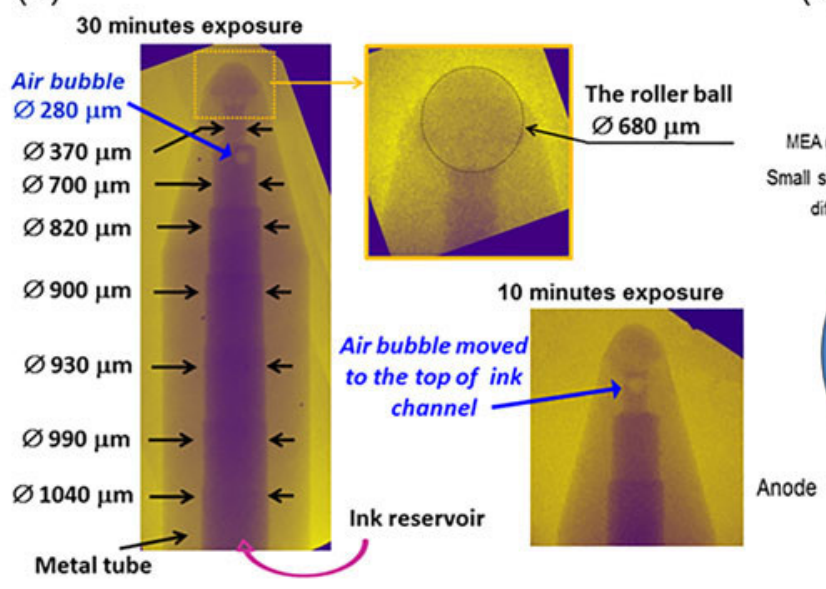

(b)

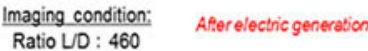
Ratio LD : 460 Exposure time: $123 \mathrm{~min}$ Detector : Lif crystal

MEA $(-50 \mu \mathrm{m})$ with diffusionlayer $(-300, \mu \mathrm{m})$ Small size FC

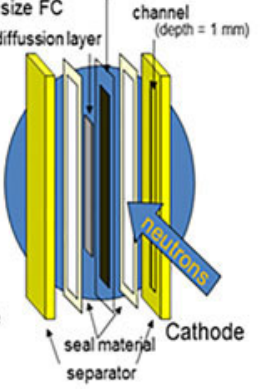

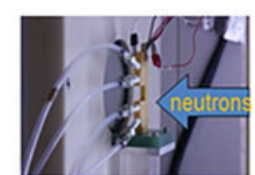
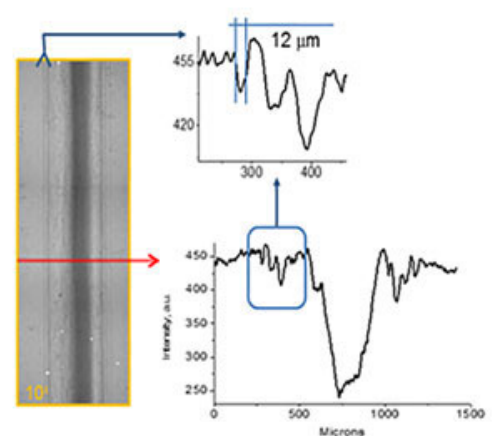

Figure 8. (a) Neutron images of a ball-point pen obtained by a tiling sequence of $4 \times$ magnified images ${ }^{[33]}$. A metal tube, a roller ball at the top and the ink in the metal tube with strong neutron attenuation are obviously distinguished. It is also clearly seen that a small air bubble of $280 \mu \mathrm{m}$ diameter in the ink has moved to the upper part of the pen between the first experiment with 30 min neutron exposure and the second measurement with 10 min exposure. (b) A schematic drawing of a small fuel cell and the neutron image of $i^{[35]}$. Tiny details with sizes of at least $\sim 12 \mu \mathrm{m}$ of the fuel cell structure and its inhomogeneity along and perpendicular to the anode-cathode directions are evidently resolved.

it is unmistakably seen how an air bubble of $\sim 280 \mu \mathrm{m}$ diameter has accidentally moved from the top of the ink channel with diameter $\sim 700 \mu \mathrm{m}$ further inside the tube in the direction of the roller bar between the two measurements (see Figure 8(a), 30 and 10 min exposure neutron images).

A very important application of micron-sized neutron radiography is obtaining detailed information about the water distribution in the membrane electrode assembly (MEA) and the gas diffusion layer (GDL) in fuel cells. As is seen from Figure 8(b), such information can be obtained by neutron irradiation of cells and registration of the image by the $\mathrm{LiF}$ crystal imaging detector ${ }^{[35]}$. A spatial resolution of better than $12 \mu \mathrm{m}$ can be undoubtedly seen along the full field of view of $\sim 1.5 \times 4.5(\mathrm{~mm})$ and allows us to distinguish the most minute details of the cell structure.

The examples presented in this section show that neutron imaging with $\mathrm{LiF}$ is suitable for the observation of detailed structures of low- $Z$ materials with high spatial resolution and dynamic range.

\section{Conclusion}

The experimental results discussed in this paper show that $\mathrm{LiF}$ crystals have excellent characteristics and great advantages compared with traditionally used neutron detectors in areas where a micron-scale spatial resolution, a high dynamic range and a high contrast are needed. Indeed, we demonstrated that the neutron images recorded with $\mathrm{LiF}$ are almost free from granular noise, and the spatial resolution reaches $\sim 5.4 \mu \mathrm{m}$; the response is highly linear to the neutron fluence with a dynamic range of at least $10^{3}$. As drawbacks, we should mention that the sensitivity of LiF to thermal neutrons is not very high and is approximately $2.5 \times$ $10^{8} \mathrm{n} / \mathrm{cm}^{2}$. At the same time, it is obvious that it could be increased by least an order of magnitude. Actually, we used natural $\mathrm{LiF}$ in our experiments, in which the concentration of ${ }^{6} \mathrm{Li}$ is not very high (the abundance is $7.4 \%$ ). However, it is expected that this sensitivity will be improved by $\sim 13$ times if we use enriched ${ }^{6} \mathrm{LiF}$ in place of natural $\mathrm{LiF}$. The sensitivity could be further improved by using polycrystalline $\mathrm{LiF}$, since poly-crystalline $\mathrm{LiF}$ coating has proved to have $\sim 10$ times higher sensitivity than LiF single crystal without degrading the resolution in soft $\mathrm{x}$-ray imaging ${ }^{[16]}$.

We hope that due to all of the abovementioned advantages of LiF crystal detectors, they will be useful not only for quantitative evaluation of various object structures in devices comprising low- $Z$ elements (including Li-ion batteries and fuel cells) but also for diagnostics of different continuous and pulsed high-intensity neutron sources, including such plasma sources as laser and z-pinch produced plasma. It is necessary to mention that the application of LiF detectors for fast neutron imaging will require a large number of neutrons. Indeed, if the cross-sections for thermal neutron interaction with $\mathrm{LiF}$ are $\sim 950$ barn (for neutron energies of tens of $\mathrm{meV}$ ), the cross-sections for interaction of DT or DD fusion neutrons with LiF crystals are lower by practically four orders of magnitude. In such a case, the intensity of the thermonuclear neutron source should be very high for practical applications of LiF imaging detectors. Meanwhile, in recent National Ignition Facility (NIF) experiments ${ }^{[39]}$ the fast neutron yield has reached the enormous value of $\sim 10^{16}$ neutrons per shot, and should be even higher in future experiments. This means that in future experiments when neutron generation reaches $\sim 10^{17}$ neutrons per shot, we could expect 
that at a distance of $50 \mathrm{~cm}$ from the target the neutron flux will be $\sim 3 \times 10^{12} \mathrm{n} / \mathrm{cm}^{2}$. Such a neutron flux should be sufficient for obtaining neutron images using a LiF crystal detector in single shot at this distance. At the same time, as was cited in Ref. [40], deuterium-tritium-loaded capsules at NIF can now regularly produce a plasma with low-energy 'ICF-thermal' neutrons with density $>10^{21}$ neutrons $\mathrm{cm}^{-3}$. This high quantity of neutrons with energy in the range of $1 \mathrm{eV}-100 \mathrm{keV}$ gives hope for successful thermal neutron imaging using $\mathrm{LiF}$ detectors due to the higher efficiency of their absorption compared with $\mathrm{MeV}$ neutrons. It is also necessary to mention that, recently, LiF films embedded with $\mathrm{Li}^{6}$ have been successfully used as converters of neutrons in tokomak experiments with thermonuclear neutrons ${ }^{[41,42]}$. The subsequent results give confidence that $\mathrm{LiF}$ detectors could be successfully used for high-resolution imaging of plasma neutron sources. Of course, direct experimental tests at laser fusion, z-pinch or tokamak installations are needed to confirm the advantages of LiF detectors for highperformance neutron imaging.

\section{Acknowledgement}

This work was partly supported by the RAS Presidium Program for Basic Research \#13 and Russian Foundation for Basic Research grant \#14-02-92107.

\section{References}

1. J. S. Brenizer, Phys. Proc. 43, 10 (2013).

2. E. H. Lehmann, P. Boillat, G. Scherrer, and G. Frei, Nucl. Instrum. Methods A 605, 123 (2009).

3. S. J. Harris, A. Timmons, D. R. Baker, and C. Monroe, Chem. Phys. Lett. 485, 265 (2010).

4. J. B. Siegel, X. Lin, A. G. Stefanopoulou, D. S. Hussey, D. L. Jacobson, and D. Gorsich, J. Electrochem. Soc. 158, A523 (2011).

5. L. G. Butler, E. H. Lehmann, and B. Schillinger, Phys. Proc. 43, 331 (2013).

6. G. P. Grim, G. L. Morgan, M. D. Wilke, P. L. Gobby, C. R. Christensen, and D. C. Wilson, Rev. Sci. Instrum. 75, 3572 (2004).

7. W. Qian, S. Qi, W. Wang, J. Cheng, and D. Liu, Rev. Sci. Instrum. 82, 093504 (2011).

8. T. Caillaud, O. Landoas, M. Briat, S. Kime, B. Rossé, I. Thfoin, J. L. Bourgade, L. Disdier, V. Yu. Glebov, F. J. Marshall, and T. C. Sangster, Rev. Sci. Instrum. 83, 033502 (2012).

9. P. Volegov, C. R. Danly, D. N. Fittinghoff, G. P. Grim, N. Guler, N. Izumi, T. Ma, F. E. Merrill, A. L. Warrick, C. H. Wilde, and D. C. Wilson, Rev. Sci. Instrum. 85, 023408 (2014).

10. K. Takahashi, S. Tazaki, J. Miyahara, Y. Karasawa, and N. Niimura, Nucl. Instrum. Methods A 377, 119 (1996).

11. H. Kobayashi, M. Satoh, and M. Matsubayashi, Nucl. Instrum. Methods A 424, 221 (1999).

12. O. H. Siegmund, J. V. Vallerga, A. S. Tremsin, J. Mcphate, and B. Feller, Nucl. Instrum. Methods A 576, 178 (2007).

13. N. Kardjilov, A. Hilger, I. Manke, M. Strobl, W. Treimer, and J. Banhart, Nucl. Instrum. Methods A 542, 16 (2005).
14. K. Nittoh, C. Konagai, T. Noji, and K. Miyabe, Nucl. Instrum. Methods A 605, 107 (2009).

15. J. H. Schulman and W. D. Compton, Color Centers in Solids (Pergamon, Oxford, 1962).

16. G. Baldacchini, S. Bollanti, F. Bonfigli, F. Flora, P. Di Lazzaro, A. Lai, T. Marolo, R. M. Montereali, D. Murra, A. Faenov, T. Pikuz, T. Lisi, G. Tomassetti, A. Reale, L. Reale, A. Ritucci, T. Limongi, L. Palladino, M. Francucci, S. Martelluci, and G. Petrocelli, Rev. Sci. Instrum. 76, 113104 (2005).

17. A. Ustione, A. Cricenti, F. Bonfigli, F. Flora, A. Lai, T. Marolo, R. M. Montereali, G. Baldacchini, A. Faenov, T. Pikuz, and L. Reale, Appl. Phys. Lett. 88, 141107 (2006).

18. Y. Fukuda, A. Faenov, T. Pikuz, M. Kando, H. Kotaki, I. Daito, J. Ma, L. M. Chen, T. Homma, K. Kawase, T. Kameshima, T. Kawachi, H. Daido, T. Kimura, T. Tajima, Y. Kato, and S. V. Bulanov, Appl. Phys. Lett. 92, 121110 (2008).

19. T. A. Pikuz, A. Ya. Faenov, S. V. Gasilov, I. Yu. Skobelev, Y. Fukuda, M. Kando, H. Kotaki, T. Homma, K. Kawase, Y. Hayahsi, T. Kawachi, H. Daido, Y. Kato, and S. V. Bulanov, Appl. Opt. 48, 6271 (2009).

20. S. V. Gasilov, A. Ya. Faenov, T. A. Pikuz, Y. Fukuda, M. Kando, T. Kawachi, I. Yu. Skobelev, H. Daido, Y. Kato, and S. V. Bulanov, Opt. Lett. 34, 3268 (2009).

21. A. Faenov, T. Pikuz, Y. Fukuda, M. Kando, H. Kotaki, T. Homma, K. Kawase, I. Skobelev, S. Gasilov, T. Kawachi, H. Daido, T. Tajima, Y. Kato, and S. Bulanov, Japan. J. Appl. Phys. 49, 06GK03 (2010).

22. L. Reale, F. Bonfigli, A. Lai, F. Flora, P. Albertano, M. L. Di Giorgio, L. Mezi, R. M. Montereali, A. Faenov, T. Pikuz, S. Almaviva, M. Francucci, P. Gaudio, S. Martellucci, M. Richetta, and A. Poma, J. Microsc. 258, 127 (2015).

23. A. Ya. Faenov, Y. Kato, M. Tanaka, T. A. Pikuz, M. Kishimoto, M. Ishino, M. Nishikino, Y. Fukuda, S. V. Bulanov, and T. Kawachi, Opt. Lett. 34, 941 (2009).

24. A. Ya. Faenov, N. A. Inogamov, V. V. Zhakhovskii, V. A. Khokhlov, K. Nishihara, Y. Kato, M. Tanaka, T. A. Pikuz, M. Kishimoto, M. Ishino, M. Nishikino, T. Nakamura, Y. Fukuda, S. V. Bulanov, and T. Kawachi, Appl. Phys. Lett. 94, 231107 (2009).

25. N. A. Inogamov, A. Ya. Faenov, V. V. Zhakhovsky, T. A. Pikuz, I. Yu. Skobelev, Yu. V. Petrov, V. A. Khokhlov, V. V. Shepelev, S. I. Anisimov, V. E. Fortov, Y. Fukuda, M. Kando, T. Kawachi, M. Nagasono, H. Ohashi, M. Yabashi, K. Tono, Y. Senda, T. Togashi, and T. Ishikawa, Contrib. Plasma Phys. 51, 419 (2011).

26. T. Pikuz, A. Faenov, A. Pirozhkov, A. Astapov, G. Klushin, S. Pikuz, Jr., N. Nagorskiy, S. Magnitskiy, T. Esirkepov, J. Koga, T. Nakamura, S. Bulanov, Y. Fukuda, Y. Hayashi, H. Kotaki, Y. Kato, and M. Kando, Phys. Status Solidi C 9, 2331 (2012).

27. T. Pikuz, A. Faenov, Y. Fukuda, M. Kando, P. Bolton, A. Mitrofanov, A. Vinogradov, M. Nagasono, H. Ohashi, M. Yabashi, K. Tono, Y. Senba, T. Togashi, and T. Ishikawa, Opt. Express 20, 3424 (2012).

28. T. Pikuz, A. Faenov, Y. Fukuda, M. Kando, P. Bolton, A. Mitrofanov, A. Vinogradov, M. Nagasono, H. Ohashi, M. Yabashi, K. Tono, Y. Senba, T. Togashi, and T. Ishikawa, Appl. Opt. 52, 509 (2013).

29. A. S. Pirozhkov, M. Kando, T. Zh. Esirkepov, P. Gallegos, H. Ahmed, E. N. Ragozin, A. Ya. Faenov, T. A. Pikuz, T. Kawachi, A. Sagisaka, J. K. Koga, M. Coury, J. Green, P. Foster, C. Brenner, B. Dromey, D. R. Symes, M. Mori, K. Kawase, T. Kameshima, Y. Fukuda, L. Chen, I. Daito, K. Ogura, Y. Hayashi, H. Kotaki, H. Kiriyama, H. Okada, N. Nishimori, T. Imazono, K. Kondo, T. Kimura, T. Tajima, H. Daido, P. Rajeev, P. McKenna, M. Borghesi, D. Neely, Y. Kato, and S. V. Bulanov, New J. Phys. 16, 093003 (2014). 
30. T. Pikuz, A. Faenov, S. Magnitskiy, N. Nagorskiy, M. Tanaka, M. Ishino, M. Nishikino, Y. Fukuda, M. Kando, Y. Kato, and T. Kawachi, High Power Laser Sci. Engng 2, e12 (2014).

31. J. J. Gilman and W. G. Johnston, J. Appl. Phys. 29, 877 (1958).

32. A. R. Lakshmanaun, U. Madhusoodanan, A. Natarajan, and B. S. Panigrahi, Phys. Stat. Sol. A 153, 265 (1996).

33. M. Matsubayashi, A. Faenov, T. Pikuz, Y. Fukuda, and Y. Kato, Nucl. Instrum. Methods Phys. Res. A 622, 637 (2010).

34. M. Matsubayashi, A. Faenov, T. Pikuz, Y. Fukuda, Y. Kato, R. Yasuda, H. Iikura, T. Nojima, and T. Sakai, Nucl. Instrum. Methods Phys. Res. A 651, 90 (2011).

35. A. Faenov, M. Matsubayashi, T. Pikuz, Y. Fukuda, M. Kando, R. Yasuda, H. Iikura, T. Nojima, T. Sakai, M. Shiozawa, and Y. Kato, Phys. Status Solidi C 9, 2231 (2012).

36. R. Yasuda, M. Matsubayashi, T. Sakai, T. Nojima, H. Iikura, M. Katagiri, K. Takano, T. Pikuz, and A. Faenov, Phys. Proc. 43, 196 (2013).

37. G. F. Knoll, Radiation Detection and Measurement (John Wiley, New York, 2000).

38. M. Matsubayashi, H. Kobayashi, T. Hibiki, and K. Mishima, Nucl. Technol. 132, 309 (2000).
39. O. A. Hurricane, D. A. Callahan, D. T. Casey, P. M. Celliers, C. Cerjan, E. L. Dewald, T. R. Dittrich, T. Döppner, D. E. Hinkel, L. F. Berzak Hopkins, J. L. Kline, S. Le Pape, T. Ma, A. G. MacPhee, J. L. Milovich, A. Pak, H.-S. Park, P. K. Patel, B. A. Remington, J. D. Salmonson, P. T. Springer, and R. Tommasini, Nature 506, 343 (2014).

40. L. A. Bernstein, D. L. Bleuel, J. A. Caggiano, C. Cerjan, R. J. Fortner, J. Gostic, P. M. Grant, N. Gharibyan, C. Hagmann, R. Hatarik, E. A. Henry, D. Sayre, D. H. G. Schneider, W. Stoeffl, D. A. Shaughnessy, D. P. McNabb, C. B. Yeamans, N. P. Zaitseva, J. A. Brown, B. H. Daub, N. M. Brickner, P. F. Davis, B. L. Goldblum, K. A. Van Bibber, J. Vujic, M. S. Basunia, R. B. Firestone, A. M. Hurst, and A. M. Rogers, Plasma Fusion Res. 9, 4404101 (2014).

41. M. Angelone, D. Lattanzi, M. Pillon, M. Marinelli, E. Milani, A. Tucciarone, G. Verona-Rinati, S. Popoviche, R. M. Montereali, M. A. Vincenti, and A. Murari, Nucl. Instrum. Methods A 595, 616 (2008).

42. S. Almaviva, M. Marinelli, E. Milani, G. Prestopino, A. Tucciarone, C. Verona, G. Verona-Rinati, M. Angelone, D. Lattanzi, M. Pillon, R. M. Montereali, and M. A. Vincenti, J. Appl. Phys. 103, 054501 (2008). 